\title{
Effects of calorie restriction on life span of microorganisms
}

\author{
Craig Skinner $\cdot$ Su-Ju Lin
}

Received: 25 June 2010 /Revised: 3 August 2010 / Accepted: 3 August 2010 /Published online: 19 August 2010

(C) The Author(s) 2010. This article is published with open access at Springerlink.com

\begin{abstract}
Calorie restriction (CR) in microorganisms such as budding and fission yeasts has a robust and welldocumented impact on longevity. In order to efficiently utilize the limited energy during $\mathrm{CR}$, these organisms shift from primarily fermentative metabolism to mitochondrial respiration. Respiration activates certain conserved longevity factors such as sirtuins and is associated with widespread physiological changes that contribute to increased survival. However, the importance of respiration during CR-mediated longevity has remained controversial. The emergence of several novel metabolically distinct microbial models for longevity has enabled CR to be studied from new perspectives. The majority of CR and life span studies have been conducted in the primarily fermentative Crabtree-positive yeasts Saccharomyces cerevisiae and Schizosaccharomyces pombe, but studies in primarily respiratory Crabtree-negative yeast and obligate aerobes can offer complementary insight into the more complex mammalian response to CR. Not only are microorganisms helping characterize a conserved cellular mechanism for CR-mediated longevity, but they can also directly impact mammalian metabolism as part of the natural gut flora. Here, we discuss the contributions of microorganisms to our knowledge of CR and longevity at the level of both the cell and the organism.
\end{abstract}

Keywords Calorie restriction · Microorganisms . Mitochondrial respiration $\cdot$ Metabolism $\cdot$ Life span · Aging

\footnotetext{
C. Skinner · S.-J. Lin $(\bowtie)$

Department of Microbiology, College of Biological Sciences, University of California,

323 Briggs Hall One Shields Ave,

Davis, CA 95616, USA

e-mail: slin@ucdavis.edu
}

\section{Introduction}

Unlike in many aspects of biology, the calorie restrictionlife span connection was first established in a complicated multicellular organism (rat; McCay et al. 1989) and then adapted to a variety of both simpler eukaryotic (Roux et al. 2010) and perhaps prokaryotic microbes (Lele et al. 2008). The advantages of using simpler systems to study the highly conserved phenomenon of calorie restriction include short life span, considerable genetic plasticity, and easier manipulation of diet. While calorie restriction in worms, flies, and mammals usually entails limiting a complex food source such as bacteria or feed pellets, calorie restriction in yeasts and bacteria can be enforced by simple carbon source limitation. Reduction of glucose, which is the preferred carbon source of most microbes, has a wide spectrum of effects in the variety of established microbial life span models, ranging from nearly 100 -fold life span extension (van Diepeningen et al. 2010) to threefold life span reduction (Oliveira et al. 2008). This large diversity in CR-mediated life span is a result of the biochemical, genetic, and metabolic identity of each microorganism.

Life span measurements conducted in Saccharomyces cerevisiae, the first microbe to gain popularity as a model of aging, have been adapted or modified to address life span in newer microbial aging models, such as Schizosaccharomyces pombe, Kluyveromyces lactis, Candida albicans, and Escherichia coli. One of the two mainstay life span assays in S. cerevisiae, chronological life span (CLS), measures how long cells can remain viable in a non-dividing state (such as in media exhausted of nutrients; Fabrizio et al. 2003). CLS can be addressed in most current microbial aging models. Measuring the total division potential of an individual cell, or replicative life span (RLS), is greatly simplified in $S$. cerevisiae due to gross morphological 
differences between mother and daughter cell (Mortimer and Johnston 1959). RLS measurement in organisms whose division is morphologically symmetric such as $S$. pombe or $E$. coli is ponderous by comparison, but studies have determined that these organisms have division asymmetries that cumulate after repeated divisions, and therefore these have a replicative life span as well (Barker and Walmsley 1999; Stewart et al. 2005). Certain attributes of new microbial aging models, such as filament senescence in Podospora anserina and differentiation in Caulobacter crescentus, provide novel methods to determine life span in these unique organisms. Calorie restriction, which extends RLS and CLS in S. cerevisiae, provides an example of longevity pathway overlap. However, augmentation or reduction of certain proteins/longevity factors linked to signaling during CR can modulate RLS and CLS differently. The pathways, proteins, and metabolism involved in the initiation or regulation of the CR-mediated longevity in assorted microbial model organisms will be addressed in this review.

\section{Crabtree-positive yeasts as microbial CR models}

The diversity of novel yeast and bacterial models for calorie restriction and longevity allows for new metabolic comparisons. Particularly interesting are those among Crabtreepositive yeasts, such as $S$. cerevisiae and $S$. pombe, Crabtree-negative yeasts, such as $K$. lactis and C. albicans, and obligate aerobe yeast such as $P$. anserina. The Crabtree effect, coined for Dr. Herbert Grace Crabtree (Crabtree 1929), refers to inhibition of aerobic metabolism when the preferred carbon source, glucose, is available. This inhibition occurs in the presence or absence of oxygen, and the term is not specific to yeasts: many mammalian tumor cells display a Crabtree effect as well (De Deken 1966; Golshani-Hebroni and Bessman 1997). Crabtree-positive yeasts prefer fermentation and actively inhibit aerobic respiration under normal circumstances: they can maintain a pool of stored extracellular carbon and enjoy a selective advantage due to other organisms' sensitivity to ethanol. Crabtree-negative yeasts should not be confused with obligate aerobes: Crabtree-negative yeasts can perform fermentation but generally only choose this form of metabolism when living anaerobically. In Crabtreenegative yeasts, there is no inhibition of aerobic respiration in the presence of glucose, and thus aerobic respiration represents the vast majority of cellular metabolism, regardless of carbon source. Obligate aerobe yeasts cannot ferment and only respire aerobically, providing another category of metabolic diversity. A further metabolic division among microbial model organisms for longevity is prokaryotes and eukaryotes. The mitochondria is the site of the most effective form of energy harvesting (respiration) in both microbial and multicellular eukaryotes, and there is considerable crosstalk between cell and mitochondria to coordinate the demand for ATP, iron-sulfur clusters, and certain amino acids (Liu and Butow 2006; Xu and Moller 2008; Zelenaya-Troitskaya et al. 1995; Veatch et al. 2009). Bacteria, however, are their own energy powerhouses, so energy coordination is less complicated. Prokaryotic model organisms such as E. coli and C. crescentus therefore provide mitochondria-independent insight into the mechanisms of $\mathrm{CR}$ (or at least information independent of mitochondria-to-cell signals). Similar to microbial eukaryote models, prokaryote models come in a variety of flavors, including facultative anaerobes (E. coli) and obligate aerobes $(C$. crescentus $)$. A brief summary of aforementioned microbes and their diverse metabolic responses to CR is shown in Fig. 1.

\section{Saccharomyces cerevisiae}

The quintessential microbial aging model, S. cerevisiae, is a commonly used organism for life span and calorie restriction studies for a multitude of different reasons. For one, mother-daughter cell asymmetry in $S$. cerevisiae is easily observed under the microscope, allowing development of the RLS assay, the first method to measure life
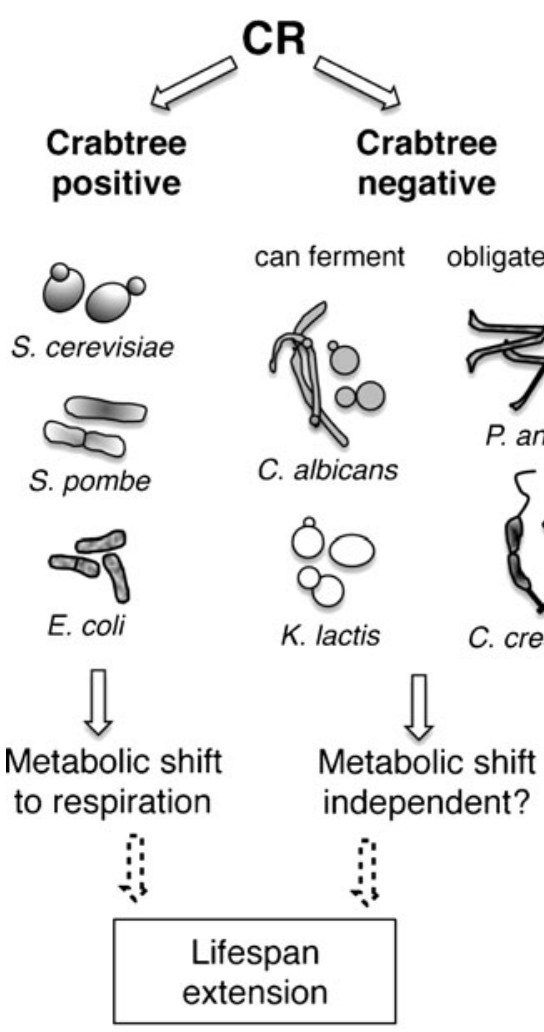

Fig. 1 A brief summary of various microbes and their diverse metabolic responses to $\mathrm{CR}$ 
span in microbes (Mortimer and Johnston 1959). Wellestablished molecular genetic techniques (recombination), genome sequence availability, and a prokaryotic-like lack of introns made $S$. cerevisiae a popular model organism for a multitude of different processes and assisted in giving budding yeast a head start in the nascent field of longevity studies. Following soon after, S. cerevisiae was also the vanguard microbe for the study of CLS (Fabrizio et al. 2003). Although RLS and CLS are addressing two very different forms of longevity, CR is a uniting factor: both RLS and CLS are extended dramatically by moderate $(0.5 \%$ glucose $)$ or severe $(0.05 \%$ glucose $)$ CR. How CR mediates life span extension, however, differs between life span measurements.

As a Crabtree-positive yeast, $S$. cerevisiae is able to repress mitochondrial oxidative respiration machinery when its preferred carbon source, glucose, is plentiful. In lieu of respiring, when glucose is abundant in its growth media, $S$. cerevisiae ferments glucose to ethanol, promoting the buildup of a metabolic by-product which it is highly tolerant of, while eliminating harmful acidic intermediates like acetic acid or lactic acid, which is the end product of mammalian fermentation. The ethanolic fermentation pathway employed by Crabtree-positive yeasts like $S$. cerevisiae and $S$. pombe has the ancillary benefits of both rapidly conferring a selective advantage against bacteria and providing a form of extracellular energy storage that not many other organisms can utilize. When glucose is scarce, such as during CR, S. cerevisiae focuses on a more efficient form of energy metabolism, mitochondrial respiration (Lin et al. 2002). This switch from fermentation to respiration is the driving force behind many physiological changes associated with longevity, although some aspects of CR appear to be independent of respiration, as illustrated by mutants in S. cerevisiae and Crabtree-negative and obligate aerobic organisms (Kaeberlein et al. 2005a; van Diepeningen et al. 2010). Development of many genetic mimics of CR, such as strains where pro-growth glucose-regulated kinases have been deleted, may provide further insight into the molecular mechanism of CR. Among those reported CR genetic mimics, strains with reduced target of rapamycin (TOR; Kaeberlein et al. 2005b), protein kinase A (PKA; Lin et al. 2000; Longo 2003), or Sch9 (an S6 kinase and AKT homolog; Longo 2003; Kaeberlein et al. 2005b; Easlon et al. 2007) activity have both increased RLS and CLS and thus closely resemble CR life span phenotypes. TOR kinase is pro-growth in the presence of certain amino acids and activates ribosomal production and proliferation while inhibiting stress resistance, mitochondrial respiration, and stationary-phase entry (Wullschleger et al. 2006; Schieke and Finkel 2007; Powers et al. 2006). PKA is activated in response to glucose through the G-protein Ras $1 / 2$ and adenylate-cyclase-mediated production of cyclic AMP (Lin et al. 2000; Longo 2003). Like TOR kinase, PKA is progrowth and upregulates cell proliferation while preventing stationary-phase entry and aerobic respiration (Portela and Moreno 2006). However, unlike moderate CR $(0.5 \%$ glucose), reduction of PKA, deletion of Sch9, or inhibition of TOR via rapamycin result in a mild to severe growth defect, and this growth defect may be contributing to longevity in either or both life span measurements, perhaps in a CR-independent fashion. There is a mammalian precedent for this: dwarf mice tend to live longer than their larger littermates (Bartke et al. 2001).

The delay in replicative aging caused by $\mathrm{CR}$ in $S$. cerevisiae is strongly linked to a number of associated factors. An increase in respiration causes a concomitant decrease in nicotinamide adenine dinucleotide, reduced form (NADH), which is a competitive inhibitor of enzymes that require oxidized nicotinamide adenine dinucleotide, oxidized form $\left(\mathrm{NAD}^{+}\right)$as a cofactor (Lin et al. 2004). Many enzymes, particularly metabolic ones, require $\mathrm{NAD}^{+}$as a cofactor, but the ones most closely associated with CR and life span are the sirtuins, characterized by the founding member in $S$. cerevisiae, silent information regulator 2 (Sir2; Lu and Lin 2010). Sirtuins are $\mathrm{NAD}^{+}$-dependent protein deacetylases: the activity $\operatorname{Sir} 2$ is best known for the deacetylation of histones H3 and H4. During CR, a decrease in cellular and nuclear NADH, enforced by a metabolic shift to mitochondrial respiration, results in increased or stabilized Sir2 activity. A primary target of Sir2 in S. cerevisiae replicative aging is the tandem ribosomal DNA (rDNA) repeats in the nucleolus. In a Sir2 deletion strain, acetylated histones are relaxed from nucleolar rDNA, allowing recombination machinery to produce extrachromosomal rDNA plasmid-like DNA circles (ERCs). These rDNA circles, or ERCs, are semi-autonomous: they can replicate on their own, like plasmids, and gradually accumulate during aging (Sinclair and Guarente 1997; Lu and Lin 2010). Sir2 does not only prevent ERC formation, it also regulates asymmetrical inheritance of ERCs and other aggregates during cell division (Erjavec and Nystrom 2007). Deletion of the replication fork block protein Fob1 results in much lower rDNA recombination and ERC formation and extends life span considerably (Defossez et al. 1999). However, rDNA repeats are not the only cellular target for Sir2: Sir2 also silences chromatin in the mating loci and telomeric and subtelomeric regions (Moazed et al. 1997; Xu et al. 2007). Since ERCs are not found in many other model organisms for aging but Sir2 orthologs are, the main effect of Sir2 in these other aging models may be due to telomeric or subtelomeric silencing (Dang et al. 2009). Asymmetrical inheritance is also a critical aspect of CR-mediated replicative longevity: if $S$. cerevisiae cells cannot restrict damaged or aggregate proteins and ERCs exclusively to the mother cell, daughter cells will have decreased RLS. In sir $2 \Delta$ mutant 
strains, RLS is dramatically reduced with or without CR treatment, but there is some controversy as to whether Sir2 can function independently of $\mathrm{CR}$ in replicative aging (Kaeberlein and Powers 2007). It is possible that CR decreases the abundance of damaged or aggregated proteins in part by Sir2-independent means but reduces rDNA recombination through Sir2. This explanation may help resolve the controversial role of Sir2 in CR and RLS and is supported by studies in Crabtree-negative yeasts and bacteria.

A metabolic shift from fermentation to respiration appears to be responsible for long CLS in calorierestricted cells as well. Similarly, respiration is required for CLS extension by reduced-growth pathway activity (Lavoie and Whiteway 2008). In contrast to replicative aging, cellular stresses during chronological aging include cell intrinsic factors, such as hydrogen peroxide and superoxide, and cell extrinsic factors, such as acetic acid and ethanol (Fabrizio et al. 2005; Magherini et al. 2009; Burtner et al. 2009; Takeda et al. 2010). Reduction of PKA, TOR, and Sch9 activity confers considerable CLS extension and, like CR, increases both respiration and cellular resistance to a variety of stresses (Bonawitz et al. 2007; Lavoie and Whiteway 2008; Pan and Shadel 2009). Oxidative scavenging contributes to chronological longevity: overexpressions of both cytosolic and mitochondrial superoxide dismutases together results in extended CLS (Fabrizio et al. 2004). The role of Sir2 in CLS is even more enigmatic than in RLS. Although sirtuin activity is required for $0.5 \%$ glucose $\mathrm{CR}$ in RLS, Sir2 actually has an inhibitory effect upon the CLS of several long-lived CRmimicking mutants (sch9 $\Delta$ and a transposon-disrupted adenylate cyclase mutant, which has low PKA activity), and deletion of Sir2 confers a minimal CLS extension (Fabrizio et al. 2005). Since during CR S. cerevisiae cells primarily respire, ultimately producing the harmless byproducts $\mathrm{CO}_{2}$ and $\mathrm{H}_{2} \mathrm{O}$, ethanol does not accumulate at high concentrations in the medium. Ethanol, while not being particularly toxic on its own, can contribute to chronological aging either by later conversion to acetic acid (Burtner et al. 2009) or on its own accord (Fabrizio et al. 2005). Additionally, mitochondrial stability is a major contributor to chronological longevity in $S$. cerevisiae. Mitochondrial stability and CLS can be increased by oxidative scavenger activity, especially those that reside in the mitochondria such as mitochondrial superoxide dismutase (Sod2; Harris et al. 2003). Not surprisingly, CR is a potent activator of both the oxidative and general stress response (Wang et al. 2009). Although the majority of cellular reactive oxygen species (ROS) are produced by mitochondrial respiration, calorie-restricted cells possess a lower concentration of ROS than untreated cells (Barros et al. 2004; Wang et al. 2009): this is likely the result of upregulation of the oxidative stress response. Programmed cell death (PCD) is also linked to mitochondrial function, both in mammals and $S$. cerevisiae, and contributes to chronological aging (Herker et al. 2004). Since singlecelled budding yeast seems to live in an environment of pure everyone-for-itself competition, there would seem to be no reason for a programmed cell death routine in $S$. cerevisiae. There is speculation that $\mathrm{PCD}$, like aging, contributes to the rejuvenation of a yeast culture by eliminating damaged cells, cells with dysfunction mitochondria, and cell which have undertaken a process which they are incapable of completing, such as mating (Hamann et al. 2008). In the case of extremely short-lived mutants, such as respiration-defective strains lacking cytochrome c1, mitochondria become punctuate and destabilized as early as the diauxic shift (Easlon et al. 2007; Skinner and Lin, data not shown). Interestingly, Rho ${ }^{0}$ S. cerevisiae strains (cells which have lost their mitochondrial DNA (mtDNA)) actually exhibit slower kinetics of chronological aging than WT cells, despite their inability to respire (Mazzoni et al. 2005). Surprisingly, S. cerevisiae shares apoptotic pathway components with mammals. Budding yeast possess a proapoptotic caspase (Yca1), which, when deleted, extends CLS (Herker et al. 2004). Additionally, PCD can be attenuated in $S$. cerevisiae by overexpressing the antiapoptotic mammalian Bcl2 (Longo et al. 1997). Calorie restriction may contribute both to mitochondrial efficiency, through upregulation of mitochondrial electron transport chain components, and mitochondrial stability, through optimization of oxidative scavengers like superoxide dismutases and catalases. With stable and necessary mitochondria, PCD can be prevented, resulting in extended chronological longevity during CR.

Overall, the contributions of $S$. cerevisiae to the studies of $\mathrm{CR}$ and aging have been considerable and have helped pave the way for further research in metazoans and other microbial model organisms. Not only did S. cerevisiae help define aging measurements in other microbes, but the characterization of Sir2, mitochondrial respiration, and TOR, PKA, and Sch9 growth kinases in CR-mediated life span all originated from the budding yeast. However, certain aspects of $S$. cerevisiae make parallels with metazoans difficult, but collaboration with the many other microbial models for aging that have been developed recently may greatly aid in resolving the microbial mechanisms behind CR.

\section{Schizosaccharomyces pombe}

Another Crabtree-positive and well-studied, both genetically and metabolically, yeast, $S$. pombe, probably has the distinction of being the second most popular microbial aging model. Like $S$. cerevisiae, aging factors such as pro- 
growth kinases and sirtuins are highly conserved between $S$. pombe and mammals. Certain characteristics including characterized RNAi machinery and non-essential protein kinase A make $S$. pombe longevity pathways distinct. Differences like these make $S$. pombe a valuable complement to $S$. cerevisiae for the study of life span and cellular aging.

One overwhelming benefit of studying $S$. cerevisiae in preference to $S$. pombe is gross morphological asymmetry between mother and daughter cells during cell division. This makes RLS measurements pedestrian in S. cerevisiae and exhausting in $S$. pombe. However, fission yeast does have a finite RLS and demonstrates division asymmetry analogous to S. cerevisiae (Erjavec et al. 2008). The effects of CR upon the RLS of $S$. pombe are currently unknown, but studies are likely already underway.

Chronological life span, on the other hand, is more thoroughly characterized in fission yeast. CR in $S$. pombe, as a threefold or 40-fold reduction in glucose, extends CLS dramatically and enhances cellular resistance to heat and peroxide (Chen and Runge 2009). Similarly, deletion of PKA (Pka1; which is not lethal in S. pombe) or the AKT homolog Sck2 resulted in an increased CLS, and deletion of both Pka1 and Sck2 extended CLS longer than either deletion alone (Roux et al. 2006), suggesting that the two kinases function in complementary or partially overlapping pathways to regulate CLS. Calorie restriction during CLS has been suggested to function in $S$. pombe by both inhibition of PKA signaling and activation of mitogenactivated protein (MAP) kinase, Sty1. Indeed, CR in rich medium (yeast extract) cannot extend CLS in fission yeast without Sty1 (Zuin et al. 2010). In contrast to parallel studies in $S$. cerevisiae, although oxygen consumption was also increased in calorie-restricted cultures (indicating higher mitochondrial respiration and a metabolic shift), CR increased intracellular ROS concentration, which may play a role in activating Sty1. However, CLS measurements may not be directly comparable between budding and fission yeasts. It should be noted that yeast-extract-based rich media contains autolyzed $S$. cerevisiae extract and therefore may not be as optimal a growth medium for fission yeast as it is for budding yeast. In addition, $S$. pombe has a shorter CLS on yeast-extract-based rich media than on minimal media, again dissimilar to $S$. cerevisiae.

Although Sir2 may negatively regulate CLS in certain mutant strains in $S$. cerevisiae, there is no evidence yet that Sir2 functions similarly in $S$. pombe. In fact, in the absence of stress attenuation proteins glutathione (Gsh1) and copper/zinc cytosolic superoxide dismutase (Sod1), deletion of Sir2 reduces CLS even further, although the doubledeletion $g \operatorname{sh} 1 \Delta \operatorname{sod} 1 \Delta$ strain is short-lived to begin with (Mutoh and Kitajima 2007). This seems to suggest that Sir2 is fulfilling an additional function in $S$. pombe, regulating certain aspects of the stress response. However, characterization of Sir2 in fission yeast remains incomplete: although it is required for certain aspects of asymmetrical protein segregation, its impact upon CR in $S$. pombe RLS is yet unknown and its effects upon AKT homologs are untested.

Life span studies in $S$. pombe have already uncovered several important aspects of the CR response in Crabtreepositive organisms, including the function of MAP and AKT kinases in CLS. We eagerly await further revelations from fission yeast, perhaps including the characterization of Sir2 in the other well-known Crabtree-positive model of aging.

\section{Crabtree-negative yeasts, facultative anaerobes, and obligate aerobes as microbial CR models}

What distinguishes Crabtree-negative yeasts and bacteria such as E. coli and C. crescentus is their inability to inhibit respiration in the presence of glucose in favor of fermentation. Since these organisms primarily respire under normal life span assay conditions, CR does not activate a fermentation-to-respiration switch as in Crabtree-positive yeasts. Crabtree-negative (and obligate aerobe) yeasts therefore provide a unique platform to study the respiration switch-independent mechanisms of CR. Perhaps these respiration switch-independent mechanisms can be even further explored using obligate aerobes like $P$. anserina and C. crescentus.

\section{Kluyveromyces lactis}

An ascomycetus budding yeast found in milk, $K$. lactis, has well-characterized genetics and is heavily studied for biological and industrial reasons. Unlike the Crabtreepositive yeasts, CR (by fourfold glucose limitation, $0.5 \%$ glucose) induces a remarkably dramatic reduction of CLS (up to threefold) and does not alter NADH-cytochrome c reductase activity or respiration (Oliveira et al. 2008). Response to CR by a metabolic switch, which is critical to chronological longevity in Crabtree-positive yeasts, is absent in $K$. lactis, as is the prolonged CLS. $K$. lactis therefore complements studies on Crabtree-positive yeast, highlighting the importance of the CR-induced fermentation-torespiration switch. However, despite its nature as an asymmetrically dividing budding yeast, RLS on $K$. lactis has not been reported. It would be interesting to note if CR can extend RLS but not CLS and if CR can induce a metabolic shift in this organism during replicative aging.

\section{Candida albicans}

More commonly known for its role in nosocomial, vaginal, and oral infections, $C$. albicans has recently also been 
established as a Crabtree-negative microbial model for aging. Although C. albicans prefers respiration to fermentation in the presence of oxygen, this organism has an atypical response to glucose concentration: higher concentrations of glucose increases resistance to certain stresses (Rodaki et al. 2009), whereas restriction of glucose increases stress resistance in $S$. cerevisiae (Wang et al. 2009) and S. pombe (Zuin et al. 2010). C. albicans, which is normally benign intestinal microflora, exists in one of two diverse states: as single cells or as invasive filaments. This organism is amenable to RLS analysis, and both the single-celled and filamentous forms have similar RLSs (Fu et al. 2008). Seemingly, in contrast to K. lactis, CR administered to C. albicans extends RLS by up to $33 \%$, and increased dosage of Sir2 extends RLS as well. To date, only RLS has been studied in C. albicans. A thorough analysis of both life span measurements could be more enlightening than one on its own, and $C$. albicans, like $K$. lactis, seems amenable to both RLS and CLS.

\section{Podospora anserina — an obligate aerobe aging model}

Despite its complex filamentous nature, $P$. anserina holds many firsts in microbial aging studies. Its characteristic hyphal senescence phenotype, described nearly 60 years ago (Rizet 1953), combined with ease of culture and genetics, laid the groundwork for its continued development as a fungal aging model. Plate-cultured $P$. anserina hyphae grow radially to a certain length; then the expanding hyphal tips begin to swell, branch abnormally, and finally rupture, causing the culture to cease growth. Eventually, the entire mycelium becomes unable to produce new filaments (infertile) as well. Although conventional life span measurements (RLS and CLS) are complicated in filamentous fungi, the unusual senescent phenotype allows for other life span measurements. For example, the length of mycelium may correspond loosely to RLS, and the time the culture can remain fertile (reproductive life span) may be comparable to CLS. CR, manifested as moderate $(0.2 \%$ glucose, 10 -fold reduction) or severe $(0.02 \%$ glucose, 100 fold reduction), results in a dramatic increase in both mycelia size before senescence and period of fertility (van Diepeningen et al. 2010).

The mitochondrial toolkit of $P$. anserina includes an electron transport chain similar to that in $S$. cerevisiae, but with two key differences. Complex I in P. anserina is a proton pump, like complex I in metazoans, but different from aforementioned single-protein mitochondrial NADH dehydrogenase in $S$. cerevisiae. Secondly, $P$. anserina possesses an alternative oxidase (AOX), which receives electrons from ubiquinone and bypasses both complexes III and IV to deliver electrons directly to molecular oxygen. Utilizing the proton-pumping complex I and AOX, an electron can flow down the electron transport chain with a minimum amount of energy harvested (one proton), but much less potential ROS production, since complex III is a major site of ROS generation. Deletion of Cox 5 or Cyc1, required subunit of complex IV and cytochrome C, respectively, results in an enormous life span extension (both reproductive and mycelia size), suggesting that either activation of AOX can promote life span or cytochromemediated respiration itself is the cause of aging and senescence in $P$. anserina (Scheckhuber and Osiewacz 2008). Recent studies indicate that constitutive activating mutations in AOX transcription factors Rse2 and Rse3 can reduce or eliminate the long life span conferred by $\operatorname{cox} 5 \Delta$ or cycl $1 \Delta$ deletions (Sellem et al. 2009). Additionally, it has been shown that overexpression of AOX protein not only suppresses the long life span of Cox 5 deletion mutants but also restores senescence (Lorin et al. 2006). In light of this new information, it would appear that the halt of respiration, not the activation of AOX, is responsible for the dramatic lengthening of life span by $\operatorname{cox} 5 \Delta$ or $\operatorname{cyc} 1 \Delta$ deletion. The link between $\mathrm{CR}$ and mitochondrial respiration, which is well-documented in $S$. cerevisiae, has not been thoroughly addressed in P. anserina at present, but preliminary studies indicate that $\mathrm{CR}$ and severe $\mathrm{CR}$ can extend life span in AOX deletion mutants (mutant strains defective in AOX activity; van Diepeningen et al. 2010). It would be interesting to note if CR can extend or further extend life span in a $\operatorname{cox} 5 \Delta$ or $\operatorname{cyc} 1 \Delta$ mutant, given the much clearer relationship between life span and respiration in $P$. anserina's distant cousin, $S$. cerevisiae. CR in $P$. anserina does lower the production of ROS, similar to $S$. cerevisiae, and optimized ROS scavenging may be a contributing factor to the life span increase during CR.

Suggested "senescence factors" for $P$. anserina myceliums include mtDNA instability, unchecked mitochondrial production of ROS, and changes in mitochondrial morphology. Accumulation of damaged or rearranged mtDNA, particularly of the well-characterized intron $\alpha$, is evident at senescent hyphal bud tips and greatly reduced in severely calorie-restricted $P$. anserina hyphae (van Diepeningen et al. 2010). Mitochondrial production of ROS is reduced by $\mathrm{CR}$ or severe $\mathrm{CR}$ as well, as mentioned earlier, and mitochondria in CR- or severe-CR-treated mycelia arrange in healthy tubular structures, in contrast to the punctuate form of senescent non-CR bud tips. CR and severe CR therefore appear to contribute to the alleviation of all three of these "senescence factors" and, unlike Cox 5 or Cyc1 deletions, do not result in sterility or require AOX for viability. $\mathrm{CR}$ in $P$. anserina, as an obligate aerobe, cannot initiate a fermentation-to-respiration metabolic switch, so the dramatic life span extension conveyed by $\mathrm{CR}$ and severe CR must originate from elsewhere. A full mechanistic explanation of $\mathrm{CR}$ in $P$. anserina, outside of an 
incomplete connection to mitochondrial ROS production and increased mtDNA stability and mitochondrial morphology, is still unavailable. However, this organism, as one of the very few obligate aerobe eukaryotic fungal models of aging, may contribute significantly to our knowledge of the fermentation-to-respiration metabolic-shift-independent events initiated by calorie restriction.

Escherichia coli-a bacterial facultative anaerobe aging model

Although $S$. pombe cell division is morphologically symmetrical, segregation of proteins, lipids, and organelles into mother and daughter cells is dissimilar at the molecular level. S. pombe therefore provides a eukaryotic example of aging in symmetrical organisms. Studies on E. coli also demonstrate that replicative aging occurs in symmetrically dividing bacteria as well and additionally proves that aging is not wholly dependent upon mitochondria. During division of the rod-shaped bacterium, the old and new copies of bacterial chromosome migrate and bind to opposite poles of the dividing cell before cytokinesis. By following the old "mother cell" pole, replicatively aged cells (more than six divisions) show a reduced growth rate and an increased mortality. Asymmetrical distribution of protein aggregates correlates with aging in E. coli: aggregates tend to migrate toward the old "mother cell" pole and are thereby excluded from the daughter cell (Lindner et al. 2008). In yeast models, aging has been associated with symmetrical cell division, and CR enhances the mother cell's ability to retain damaged proteins, lipids, and organelles, enhancing the fitness of her daughter. Contrastingly, CR in E. coli has recently been shown to enforce symmetrical cell division while prolonging longevity (Lele et al. 2008), although it is possible that CR simply reduces oxidative damage and protein aggregation below a detectable limit. This would mean that mother cell retention of aggregates and damage is preserved during CR, but the amount of aggregates and damaged proteins is significantly reduced in both mother and daughter cells, similar to what occurs during replicative aging in $S$. cerevisiae (ReverterBranchat et al. 2004).

Caulobacter crescentus - a bacterial obligate aerobe aging model

Yeast and bacteria with symmetrical division were thought to be immune to the effects of aging until recently. The Caulobacter life cycle begins as a motile flagellate, or "swarmer," and culminates as a reproducing sessile cell anchored to a surface with a holdfast or "stalked" cell. This unique asymmetrical division not only enables motherdaughter discrimination but also facilitates mother cell retention. Through the use of a microscopy flow chamber, the motile daughter cells can be continuously washed out while tallying the mother cell's RLS (Ackermann et al. 2003, 2007). Unlike with E. coli, the effects of CR upon Caulobacter are untested currently, but a comprehensive study would be illuminating and is likely currently underway. Information from $C$. crescentus would be complementary to RLS measurements in $K$. lactis and $C$. albicans: if the RLS of C. crescentus could be extended by $\mathrm{CR}$, this would indicate either a respiration-independent component to replicative longevity or an unforeseen metabolic shift by CR (in an obligate aerobe).

\section{Metabolic preference of $C R$ in microbes and its relevance to mammalian aging}

While mitochondrial respiration plays a role in microbial aging, there is still no clear consensus as to what exactly that role is. In the obligate aerobe $P$. anserina, inhibition of respiration can extend life span even more dramatically than CR. In addition, CR does not enforce a metabolic switch to respiration since this organism cannot ferment and has no options other than respiration. The mechanism of CR-mediated longevity is clearer for the Crabtree-positive yeasts: CR does enact a metabolic shift to respiration, so respiration could be responsible for the multitude of lifespan-extending changes during $\mathrm{CR}$ (increased resistance to stress, Sir2 activity, etc.). Since CR does not induce a metabolic shift in the Crabtree-negative yeast $K$. lactis, CR does not extend CLS, as expected. However, RLS is extended by CR in the Crabtree-negative yeast $C$. albicans. In aggregate, these studies suggest that perhaps RLS and CLS are controlled by different CR-mediated factors: respiration and metabolite production play a central role in chronological longevity, while sirtuin activation and division asymmetry have a considerable impact upon replicative longevity. Controlling the concentration of ROS and the stability of mitochondria are critical for both life span measurements, since dysfunctional mitochondria, in many of these microbial species, eventually result in cellular death or senescence.

Though metazoans are much more complicated than single-celled or filamentous microbes, microbes, especially the eukaryotic variety, are very similar to metazoans at the cellular level. Many CR and longevity-associated factors/ pathways are highly conserved from yeast to mammals, including mitochondrial respiration, Sir2 family proteins (sirtuins), pro-growth TOR and PKA signaling pathways, and metabolic pathways such as $\mathrm{NAD}^{+}$biosynthesis. $E$. coli, though lacking mitochondria and chromatin, nevertheless possess a homolog to $\mathrm{Sir} 2$ (CobB) which is important in chemotaxis and may offer insight into early 
connections between sirtuins and metabolism (Landry et al. 2000). The significance of some of these pathways in mediating beneficial effects of CR was first recognized in simple model organisms, which was later found parallel in complex higher eukaryotes. To date, the detailed mechanisms for how these longevity factors/pathways respond to $\mathrm{CR}$ and elicit diverse beneficial effects in different cell types/organisms have remained unclear. Contradictions also persist as to whether these factors indeed affect longevity by similar mechanisms in mammals. For example, whether increased or decreased mitochondrial activity is beneficial to life span and whether mitochondrial respiration is the major mediator of CR (Guarente 2008) are still highly debatable. It has been suggested that lowering the electron transport activity is beneficial for longevity (Kim 2007), which is in line with studies of cytochrome c deletion in $P$. anserina. However, inhibition of the electron transport activity is detrimental to life span in $S$. cerevisiae, which also blocks certain CR-induced effects in yeast and mammalian cells (Lin et al. 2002, 2004; Guarente 2008). In metazoans, several aging-related phenotypes have also been associated with a decline in electron transport activity (Lin and Beal 2006; Someya et al. 2007). Much clarification has yet to be done on the effects of mitochondria and other longevity factors on CR and aging. Microbial models not only provide a powerful genetic tool for the identification of critical components in CR and aging but also serve as a platform for studying these longevity factors at the biochemical/molecular level.

Which of these models more closely resemble human aging, Crabtree-positive or Crabtree-negative yeasts? Since most human cells primarily respire when oxygen is available and ferment to lactate when it is not, at first glance, it would appear that Crabtree-negative yeasts have the edge in relevance. However, calorie restriction does induce a metabolic response in mouse white adipose tissue (Nisoli et al. 2005): mitochondrial biogenesis is increased and fatty acids are more readily consumed. Perhaps this represents a change from energy storage to utilization. The CR-induced metabolic shift from ethanol production, which could be considered a form of energy storage, to aerobic respiration, which is obviously a form of energy utilization, may actually be more similar to what happens in certain human cells, like adipose tissue. Similarly, one might wonder which of these life span measurements (RLS, CLS, $P$. anserina senescence) might be the most relevant to mammalian aging. CLS measures the survival of cell in stationary phase, and the vast majority of human cells at any given time post-development are in $\mathrm{G}_{0}$. On the other hand, RLS measures division potential and would be of more use for the study of dividing cells. Cells actively dividing in mammals include germ cells and a plethora of different types of stem cells. However, while microbial mother cells sacrifice themselves for their progeny by retaining damaged and aggregated proteins or DNA, stem cells thrust accumulated damage upon their progeny, thereby keeping themselves pristine. Nevertheless, RLS may currently be the most accurate microbial parallel to stem cell aging. Therefore, a combination of the aforementioned life span measurements would probably be the best way to uncover new conserved determinants of aging. Conserved longevity factors such as the TOR, PKA kinases, and the sirtuins are excellent targets for therapeutic interventions. Rapamycin, a TOR inhibitor, has been shown to extend life span in yeast (Powers et al. 2006) and mice (Harrison et al. 2009). Resveratrol, a potent sirtuin activator, can prolong longevity in yeast and metazoans (Wood et al. 2004; Valenzano et al. 2006). As more conserved longevity factors are revealed, the potential for life-span-extending therapeutics increases, and microorganisms, due to rapid screening and life span procedures, remain at the forefront of this field.

\section{Calorie restriction and intestinal microflora}

Although microbial model organisms can assist in elucidating highly conserved mechanisms, mediating the metabolic response to $\mathrm{CR}$ in mammalian cells, microbes also play a direct role in mammalian metabolism. Outnumbering our own cells nearly 10 to 1 and our genes 100 to 1 , the human intestinal microbiome is extremely diverse, and some consider it to be the only organ "outside the body." Microbial metabolism is infinitely more complex and diverse than that of their mammal hosts, and much of what we eat cannot be digested without the aid of gut microflora. Obese mice with mutated leptin genes (ob/ob) have dramatic changes to gut microbe composition relative to lean littermates (Turnbaugh et al. 2006), but whether gut microflora changes are the cause or a symptom of obesity is still unclear (Backhed 2009). The supremacy of Bacteroides or Firmicutes bacterial phyla, which account for $90 \%$ of the species found in the gut microflora, is linked to obesity: obese mice have a significantly higher percentage of Firmicutes inhabiting their gut, whereas lean mice have fewer Firmicutes and more Bacteroides (Turnbaugh et al. 2006). It is thought that ob/ob mice and possibly other forms of obesity both encourage the proliferation of Firmicutes and have increased energy harvesting and storage potential, possibly through utilization of Firmicutes metabolic by-products. Remarkably, if the gut microbiome of an ob/ob mouse is transplanted into a lean littermate, body fat begins accumulating at a significantly higher rate. Thus, gut microbiota composition can play a significant part in energy harvesting, energy storage, and development of obesity. 
Since gut microflora composition could potentially be both an indicator of obesity or a direct therapeutic target, the field of gut microbial genomics and metabolomics has been expanding rapidly. After the aforementioned landmark studies in mice, human microflora is being studied extensively. In humans (by studying the fecum of pairs of obese or lean twins and their mothers), obese individuals not only harbored less Bacteriodes but also had less overall gut bacterial diversity (Turnbaugh et al. 2009). It is unclear whether the lower bacterial diversity in obese subjects is due to a more homogenous (high in fats, protein, and carbohydrates) diet, although a lower variety of dietary intake could result in a less diverse microbiome. Other than Firmicutes, Achaea methanogens were enriched in obese fecum (Zhang et al. 2009). Methanogens could interact metabolically with $\mathrm{H}_{2}$-producting bacteria (such as Prevotellaceae, phylum Bacteriodes), resulting in more efficient conversion of hard-to-digest fiber polysaccharides to shortchain fatty acids. Short-chain fatty acids like acetate and succinate are readily absorbed by the gut lining, unlike their complex fiber precursors. Increased absorption of shortchain fatty acids can then be stored as fat. The obesityassociated microbiome may be even more insidious and send signals for fat deposition to their host.

But what about the roles of intestinal microbiota in CR? A recent study in Labrador retrievers determined not only that CR can extend the life span of dogs, but also, in aged dogs (9 years), the quantity of certain microbial aromatic metabolites (hippurate, PAG, and 4-HPPA) in urine significantly increased relative to those fed a rich diet (Wang et al. 2007). The microbial mammalian co-metabolite hippurate has an inverse correlation with obesity; low levels of hippurate are also related to high blood pressure and type II diabetes (Calvani et al. 2010). Additionally, the levels of metabolites such as creatine, lactate, acetate, and succinate were decreased in the urine of dietary-restricted dogs. Lactate and acetate are fermentation by-products and can be absorbed through the gut lining, indicating increased bioavailability of energy. If more energy is available, possibly due to a preponderance of intestinal Firmicutes or methanogens, more energy can be stored. Aromatic metabolite production, primarily generated by bacteria, in urine by $\mathrm{CR}$ may indicate a microfloral remodeling. Reduced consumable energy provided by gut microflora leads to more efficient energy harvesting (respiration) as well as less energy storage, promoting stable mitochondrial function, and ultimately lean and long-lived hosts (Fig. 2). Naturally, it would be exciting to investigate whether treatment with any of these CR-linked microbial chemicals, like hippurate, can help induce a CR response. Failing manipulation of mammalian cells by microbial by-products, could CR microflora be transmissible, like obesity microflora in rats? If CR-related microbiota, transplanted into another animal,

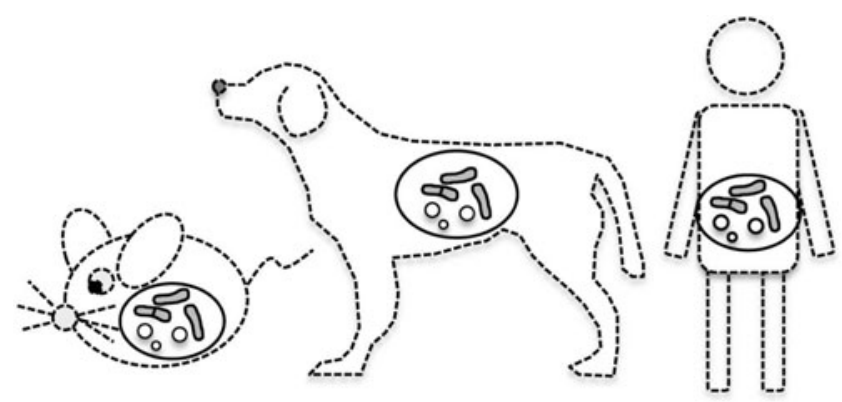

Obese

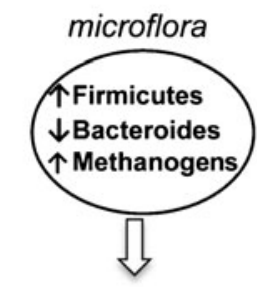

More short-chain fatty acids

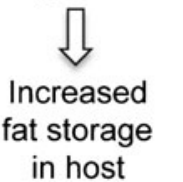

CR

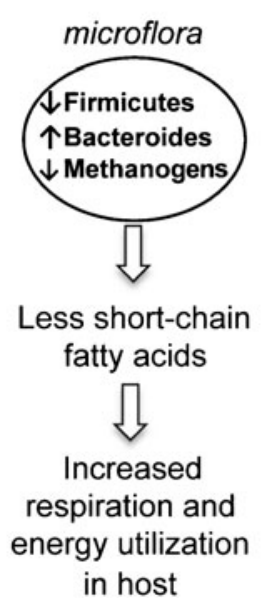

Fig. 2 Intestinal microflora may contribute to CR- and obesityinduced metabolic changes in host organisms

can induce a CR-like effect, even in the short term, this could open the door to future probiotics.

\section{Conclusion}

Insight into the mechanisms behind CR has been greatly facilitated by studying a variety of microbial model organisms. Although the importance of mitochondria and respiration in longevity is not fully understood in any organism, the combined power of metabolically distinct microbial model organisms is expanding our understanding of the respirationcalorie restriction connection. Certain life span measurements (CLS) appear to be wholly dependent upon a fermentation-torespiration metabolic shift, while others (RLS) seem to be affected by additional CR-mediated factors as well. Future studies in prokaryotes may reveal novel non-mitochondrial components of the $\mathrm{CR}$ response. Microbes are more similar to metazoans than we would expect: they share pathways for programmed cell death and all exhibit a form of aging. Study of CR and longevity in microbial models has already revealed much about aging in human cells and, due to considerable relative simplicity, remains a powerful strategy for further characterization of the $\mathrm{CR}$ response. But microbes can help us 
understand CR both within and without: not only can microbial model organisms enlighten us on how our cells age, but intestinal microbes can control our exposure to nutrients and enforce CR exogenously. In the long term, we should strive to understand how we age on a cellular level and how calorie restriction could potentially promote longevity. But in the short term, perhaps we should also look to the microbes within us.

Acknowledgments We are grateful to the researchers whose work provided the basis for this review. The Lin laboratory is supported by the National Institute on Aging (RO1-AG24351).

Open Access This article is distributed under the terms of the Creative Commons Attribution Noncommercial License which permits any noncommercial use, distribution, and reproduction in any medium, provided the original author(s) and source are credited.

\section{References}

Ackermann M, Stearns SC, Jenal U (2003) Senescence in a bacterium with asymmetric division. Science 300(5627):1920. doi:10.1126/ science. 1083532

Ackermann M, Schauerte A, Stearns SC, Jenal U (2007) Experimental evolution of aging in a bacterium. BMC Evol Biol 7:126. doi:10.1186/1471-2148-7-126

Backhed F (2009) Changes in intestinal microflora in obesity: cause or consequence? J Pediatr Gastroenterol Nutr 48(Suppl 2):S56-S57. doi:10.1097/MPG.0b013e3181a11851

Barker MG, Walmsley RM (1999) Replicative ageing in the fission yeast Schizosaccharomyces pombe. Yeast 15(14):1511-1518. doi:10.1002/(SICI)1097-0061(199910)15:14<1511::AIDYEA482>3.0.CO;2-Y

Barros MH, Bandy B, Tahara EB, Kowaltowski AJ (2004) Higher respiratory activity decreases mitochondrial reactive oxygen release and increases life span in Saccharomyces cerevisiae. J Biol Chem 279(48):49883-49888

Bartke A, Brown-Borg H, Mattison J, Kinney B, Hauck S, Wright C (2001) Prolonged longevity of hypopituitary dwarf mice. Exp Gerontol 36(1):21-28. doi:S0531-5565(00)00205-9

Bonawitz ND, Chatenay-Lapointe M, Pan Y, Shadel GS (2007) Reduced tor signaling extends chronological life span via increased respiration and upregulation of mitochondrial gene expression. Cell Metab 5(4):265-277. doi:10.1016/j.cmet.2007.02.009

Burtner CR, Murakami CJ, Kennedy BK, Kaeberlein M (2009) A molecular mechanism of chronological aging in yeast. Cell Cycle 8(8):1256-1270. doi:8287

Calvani R, Miccheli A, Capuani G, Tomassini Miccheli A, Puccetti C, Delfini M, Iaconelli A, Nanni G, Mingrone G (2010) Gut microbiome-derived metabolites characterize a peculiar obese urinary metabotype. Int J Obes (Lond) 34(6):1095-1098. doi:10.1038/ijo.2010.44

Chen BR, Runge KW (2009) A new Schizosaccharomyces pombe chronological lifespan assay reveals that caloric restriction promotes efficient cell cycle exit and extends longevity. Exp Gerontol 44(8):493-502. doi:10.1016/j.exger.2009.04.004

Crabtree HG (1929) Observations on the carbohydrate metabolism of tumours. Biochem J 23(3):536-545

Dang W, Steffen KK, Perry R, Dorsey JA, Johnson FB, Shilatifard A, Kaeberlein M, Kennedy BK, Berger SL (2009) Histone h4 lysine
16 acetylation regulates cellular lifespan. Nature 459(7248):802807. doi:10.1038/nature 08085

De Deken RH (1966) The Crabtree effect: a regulatory system in yeast. J Gen Microbiol 44(2):149-156

Defossez PA, Prusty R, Kaeberlein M, Lin SJ, Ferrigno P, Silver PA, Keil RL, Guarente L (1999) Elimination of replication block protein fob1 extends the life span of yeast mother cells. Mol Cell 3(4):447-455. doi:S1097-2765(00)80472-4

Easlon E, Tsang F, Dilova I, Wang C, Lu SP, Skinner C, Lin SJ (2007) The dihydrolipoamide acetyltransferase is a novel metabolic longevity factor and is required for calorie restriction-mediated life span extension. J Biol Chem 282(9):6161-6171

Erjavec N, Nystrom T (2007) Sir2p-dependent protein segregation gives rise to a superior reactive oxygen species management in the progeny of Saccharomyces cerevisiae. Proc Natl Acad Sci U S A 104(26):10877-10881. doi:10.1073/pnas.0701634104

Erjavec N, Cvijovic M, Klipp E, Nystrom T (2008) Selective benefits of damage partitioning in unicellular systems and its effects on aging. Proc Natl Acad Sci U S A 105(48):18764-18769. doi:10.1073/ pnas. 0804550105

Fabrizio P, Liou LL, Moy VN, Diaspro A, Valentine JS, Gralla EB, Longo VD (2003) Sod2 functions downstream of sch9 to extend longevity in yeast. Genetics 163(1):35-46

Fabrizio P, Pletcher SD, Minois N, Vaupel JW, Longo VD (2004) Chronological aging-independent replicative life span regulation by msn $2 / \mathrm{msn} 4$ and sod 2 in Saccharomyces cerevisiae. FEBS Lett 557(1-3):136-142. doi:S0014579303014625

Fabrizio P, Gattazzo C, Battistella L, Wei M, Cheng C, McGrew K, Longo VD (2005) Sir2 blocks extreme life-span extension. Cell 123(4):655-667. doi:10.1016/j.cell.2005.08.042

$\mathrm{Fu}$ XH, Meng FL, Hu Y, Zhou JQ (2008) Candida albicans, a distinctive fungal model for cellular aging study. Aging Cell 7 (5):746-757. doi:10.1111/j.1474-9726.2008.00424.x

Golshani-Hebroni SG, Bessman SP (1997) Hexokinase binding to mitochondria: a basis for proliferative energy metabolism. J Bioenerg Biomembr 29(4):331-338

Guarente L (2008) Mitochondria - a nexus for aging, calorie restriction, and sirtuins? Cell 132(2):171-176

Hamann A, Brust D, Osiewacz HD (2008) Apoptosis pathways in fungal growth, development and ageing. Trends Microbiol 16 (6):276-283. doi:10.1016/j.tim.2008.03.003

Harris N, Costa V, MacLean M, Mollapour M, Moradas-Ferreira P, Piper PW (2003) Mnsod overexpression extends the yeast chronological $(\mathrm{g}(0))$ life span but acts independently of sir2p histone deacetylase to shorten the replicative life span of dividing cells. Free Radic Biol Med 34(12):1599-1606. doi:S0891584903002107

Harrison DE, Strong R, Sharp ZD, Nelson JF, Astle CM, Flurkey K, Nadon NL, Wilkinson JE, Frenkel K, Carter CS, Pahor M, Javors MA, Fernandez E, Miller RA (2009) Rapamycin fed late in life extends lifespan in genetically heterogeneous mice. Nature 460 (7253):392-395. doi:10.1038/nature08221

Herker E, Jungwirth H, Lehmann KA, Maldener C, Frohlich KU, Wissing S, Buttner S, Fehr M, Sigrist S, Madeo F (2004) Chronological aging leads to apoptosis in yeast. J Cell Biol 164 (4):501-507. doi:10.1083/jcb.200310014

Kaeberlein M, Powers RW 3rd (2007) Sir2 and calorie restriction in yeast: a skeptical perspective. Ageing Res Rev 6(2):128-140. doi:10.1016/j.arr.2007.04.001

Kaeberlein M, Hu D, Kerr EO, Tsuchiya M, Westman EA, Dang N, Fields S, Kennedy BK (2005a) Increased life span due to calorie restriction in respiratory-deficient yeast. PLoS Genet 1(5):e69. doi:10.1371/journal.pgen.0010069

Kaeberlein M, Powers RW 3rd, Steffen KK, Westman EA, Hu D, Dang N, Kerr EO, Kirkland KT, Fields S, Kennedy BK (2005b) Regulation of yeast replicative life span by tor and sch9 in response to nutrients. Science 310(5751):1193-1196. doi:10.1126/science.1115535 
Kim SK (2007) Common aging pathways in worms, flies, mice and humans. J Exp Biol 210(Pt 9):1607-1612. doi:10.1242/jeb.004887

Landry J, Sutton A, Tafrov ST, Heller RC, Stebbins J, Pillus L, Sternglanz R (2000) The silencing protein sir2 and its homologs are NAD-dependent protein deacetylases. Proc Natl Acad Sci U S A 97(11):5807-5811. doi:10.1073/pnas.110148297

Lavoie H, Whiteway M (2008) Increased respiration in the sch9delta mutant is required for increasing chronological life span but not replicative life span. Eukaryot Cell 7(7):1127-1135. doi:10.1128/ EC.00330-07

Lele U, Belsare P, Watve S, Bari S, Karande S, Watve M (2008) Caloric restriction causes symmetric cell division and delays aging in Escherichia coli. Nat Proceedings. doi:hdl:10101/ npre.2008.2071.1

Lin MT, Beal MF (2006) Mitochondrial dysfunction and oxidative stress in neurodegenerative diseases. Nature 443(7113):787-795. doi:10.1038/nature05292

Lin SJ, Defossez PA, Guarente L (2000) Requirement of nad and sir2 for life-span extension by calorie restriction in Saccharomyces cerevisiae. Science 289(5487):2126-2128. doi:8813

Lin SJ, Kaeberlein M, Andalis AA, Sturtz LA, Defossez PA, Culotta VC, Fink GR, Guarente L (2002) Calorie restriction extends Saccharomyces cerevisiae lifespan by increasing respiration. Nature 418(6895):344-348. doi:10.1038/nature00829

Lin SJ, Ford E, Haigis M, Liszt G, Guarente L (2004) Calorie restriction extends yeast life span by lowering the level of NADH. Genes Dev 18:12-16

Lindner AB, Madden R, Demarez A, Stewart EJ, Taddei F (2008) Asymmetric segregation of protein aggregates is associated with cellular aging and rejuvenation. Proc Natl Acad Sci U S A 105 (8):3076-3081. doi:10.1073/pnas.0708931105

Liu Z, Butow RA (2006) Mitochondrial retrograde signaling. Annu Rev Genet 40:159-185. doi:10.1146/annurev.genet.40.110405.090613

Longo VD (2003) The ras and sch9 pathways regulate stress resistance and longevity. Exp Gerontol 38(7):807-811. doi: S053155650300113X

Longo VD, Ellerby LM, Bredesen DE, Valentine JS, Gralla EB (1997) Human bcl-2 reverses survival defects in yeast lacking superoxide dismutase and delays death of wild-type yeast. J Cell Biol 137(7):1581-1588

Lorin S, Dufour E, Sainsard-Chanet A (2006) Mitochondrial metabolism and aging in the filamentous fungus Podospora anserina. Biochim Biophys Acta 1757(5-6):604-610. doi:10.1016/j.bbabio.2006.03.005

$\mathrm{Lu}$ SP, Lin SJ (2010) Regulation of yeast sirtuins by $\mathrm{NAD}(+)$ metabolism and calorie restriction. Biochim Biophys Acta 1804 (8):1567-1575. doi:10.1016/j.bbapap.2009.09.030

Magherini F, Carpentieri A, Amoresano A, Gamberi T, De Filippo C, Rizzetto L, Biagini M, Pucci P, Modesti A (2009) Different carbon sources affect lifespan and protein redox state during Saccharomyces cerevisiae chronological ageing. Cell Mol Life Sci 66(5):933-947. doi:10.1007/s00018-009-8574-Z

Mazzoni C, Herker E, Palermo V, Jungwirth H, Eisenberg T, Madeo F, Falcone $\mathrm{C}$ (2005) Yeast caspase 1 links messenger rna stability to apoptosis in yeast. EMBO Rep 6(11):1076-1081. doi:10.1038/sj. embor.7400514

McCay CM, Crowell MF, Maynard LA (1989) The effect of retarded growth upon the length of life span and upon the ultimate body size. 1935. Nutrition 5(3):155-171, discussion 172

Moazed D, Kistler A, Axelrod A, Rine J, Johnson AD (1997) Silent information regulator protein complexes in Saccharomyces cerevisiae: a sir $2 / \operatorname{sir} 4$ complex and evidence for a regulatory domain in sir4 that inhibits its interaction with sir3. Proc Natl Acad Sci U S A 94(6):2186-2191

Mortimer RK, Johnston JR (1959) Life span of individual yeast cells. Nature 183(4677):1751-1752
Mutoh N, Kitajima S (2007) Accelerated chronological aging of a mutant fission yeast deficient in both glutathione and superoxide dismutase having cu and $\mathrm{zn}$ as cofactors and its enhancement by sir2 deficiency. Biosci Biotechnol Biochem 71(11):2841-2844. doi:JST.JSTAGE/bbb/70307

Nisoli E, Tonello C, Cardile A, Cozzi V, Bracale R, Tedesco L, Falcone S, Valerio A, Cantoni O, Clementi E, Moncada S, Carruba MO (2005) Calorie restriction promotes mitochondrial biogenesis by inducing the expression of enos. Science 310 (5746):314-317. doi:10.1126/science.1117728

Oliveira GA, Tahara EB, Gombert AK, Barros MH, Kowaltowski AJ (2008) Increased aerobic metabolism is essential for the beneficial effects of caloric restriction on yeast life span. J Bioenerg Biomembr 40(4):381-388. doi:10.1007/s10863-0089159-5

Pan Y, Shadel GS (2009) Extension of chronological life span by reduced tor signaling requires down-regulation of $s c h 9 p$ and involves increased mitochondrial OXPHOS complex density. Aging (Albany NY) 1(1):131-145

Portela P, Moreno S (2006) Glucose-dependent activation of protein kinase a activity in Saccharomyces cerevisiae and phosphorylation of its tpk1 catalytic subunit. Cell Signal 18(7):1072-1086. doi:10.1016/j.cellsig.2005.09.001

Powers RW 3rd, Kaeberlein M, Caldwell SD, Kennedy BK, Fields S (2006) Extension of chronological life span in yeast by decreased tor pathway signaling. Genes Dev 20(2):174-184. doi:10.1101/ gad. 1381406

Reverter-Branchat G, Cabiscol E, Tamarit J, Ros J (2004) Oxidative damage to specific proteins in replicative and chronological-aged Saccharomyces cerevisiae: common targets and prevention by calorie restriction. J Biol Chem 279(30):31983-31989. doi:10.1074/jbc.M404849200M404849200

Rizet G (1953) Longevity of strains of Podospora anserina. C R Hebd Seances Acad Sci 237(18):1106-1109

Rodaki A, Bohovych IM, Enjalbert B, Young T, Odds FC, Gow NA, Brown AJ (2009) Glucose promotes stress resistance in the fungal pathogen Candida albicans. Mol Biol Cell 20(22):48454855. doi:10.1091/mbc.E09-01-0002

Roux AE, Quissac A, Chartrand P, Ferbeyre G, Rokeach LA (2006) Regulation of chronological aging in Schizosaccharomyces pombe by the protein kinases Pka1 and Sck2. Aging Cell 5(4): 345-57. doi:10.1111/j.1474-9726.2006.00225.x

Roux AE, Chartrand P, Ferbeyre G, Rokeach LA (2010) Fission yeast and other yeasts as emergent models to unravel cellular aging in eukaryotes. J Gerontol A Biol Sci Med Sci 65(1):1-8. doi:10.1093/ gerona/glp152

Scheckhuber CQ, Osiewacz HD (2008) Podospora anserina: a model organism to study mechanisms of healthy ageing. Mol Genet Genomics 280(5):365-374. doi:10.1007/s00438-008-03786

Schieke SM, Finkel T (2007) Tor and aging: less is more. Cell Metab 5(4):233-235. doi:10.1016/j.cmet.2007.03.005

Sellem CH, Bovier E, Lorin S, Sainsard-Chanet A (2009) Mutations in two zinc-cluster proteins activate alternative respiratory and gluconeogenic pathways and restore senescence in long-lived respiratory mutants of Podospora anserina. Genetics 182(1):6978. doi:10.1534/genetics.109.100834

Sinclair DA, Guarente L (1997) Extrachromosomal rdna circles-a cause of aging in yeast. Cell 91(7):1033-1042

Someya S, Yamasoba T, Prolla TA, Tanokura M (2007) Genes encoding mitochondrial respiratory chain components are profoundly down-regulated with aging in the cochlea of $\mathrm{dba} / 2 \mathrm{j}$ mice. Brain Res 1182:26-33. doi:10.1016/j.brainres.2007.08.090

Stewart EJ, Madden R, Paul G, Taddei F (2005) Aging and death in an organism that reproduces by morphologically symmetric division. PLoS Biol 3(2):e45. doi:10.1371/journal.pbio.0030045 
Takeda K, Yoshida T, Kikuchi S, Nagao K, Kokubu A, Pluskal T, VillarBriones A, Nakamura T, Yanagida M (2010) Synergistic roles of the proteasome and autophagy for mitochondrial maintenance and chronological lifespan in fission yeast. Proc Natl Acad Sci U S A 107(8):3540-3545. doi:10.1073/pnas.0911055107

Turnbaugh PJ, Ley RE, Mahowald MA, Magrini V, Mardis ER, Gordon JI (2006) An obesity-associated gut microbiome with increased capacity for energy harvest. Nature 444(7122):10271031. doi: 10.1038 /nature 05414

Turnbaugh PJ, Hamady M, Yatsunenko T, Cantarel BL, Duncan A, Ley RE, Sogin ML, Jones WJ, Roe BA, Affourtit JP, Egholm M, Henrissat B, Heath AC, Knight R, Gordon JI (2009) A core gut microbiome in obese and lean twins. Nature 457(7228):480-484. doi:10.1038/nature 07540

Valenzano DR, Terzibasi E, Genade T, Cattaneo A, Domenici L, Cellerino A (2006) Resveratrol prolongs lifespan and retards the onset of age-related markers in a short-lived vertebrate. Curr Biol 16(3):296-300. doi:10.1016/j.cub.2005.12.038

van Diepeningen AD, Slakhorst SM, Koopmanschap AB, Ikink GJ, Debets AJ, Hoekstra RF (2010) Calorie restriction in the filamentous fungus Podospora anserina. Exp Gerontol 45(78):516-524. doi:10.1016/j.exger.2010.01.002

Veatch JR, McMurray MA, Nelson ZW, Gottschling DE (2009) Mitochondrial dysfunction leads to nuclear genome instability via an iron-sulfur cluster defect. Cell 137(7):1247-1258. doi:10.1016/j.cell.2009.04.014

Wang Y, Lawler D, Larson B, Ramadan Z, Kochhar S, Holmes E, Nicholson JK (2007) Metabonomic investigations of aging and caloric restriction in a life-long dog study. J Proteome Res 6 (5):1846-1854. doi:10.1021/pr060685n
Wang C, Skinner C, Easlon E, Lin SJ (2009) Deleting the 14-3-3 protein bmh1 extends life span in Saccharomyces cerevisiae by increasing stress response. Genetics 183(4):1373-1384. doi:10.1534/genetics.109.107797

Wood JG, Rogina B, Lavu S, Howitz K, Helfand SL, Tatar M, Sinclair D (2004) Sirtuin activators mimic caloric restriction and delay ageing in metazoans. Nature 430(7000):686-689

Wullschleger S, Loewith R, Hall MN (2006) Tor signaling in growth and metabolism. Cell 124(3):471-484. doi:10.1016/j.cell. 2006.01.016

$\mathrm{Xu}$ XM, Moller SG (2008) Iron-sulfur cluster biogenesis systems and their crosstalk. Chembiochem 9(15):2355-2362. doi:10.1002/ cbic. 200800384

Xu F, Zhang Q, Zhang K, Xie W, Grunstein M (2007) Sir2 deacetylates histone h3 lysine 56 to regulate telomeric heterochromatin structure in yeast. Mol Cell 27(6):890-900. doi:10.1016/j.molcel.2007.07.021

Zelenaya-Troitskaya O, Perlman PS, Butow RA (1995) An enzyme in yeast mitochondria that catalyzes a step in branched-chain amino acid biosynthesis also functions in mitochondrial DNA stability. EMBO J 14(13):3268-3276

Zhang H, DiBaise JK, Zuccolo A, Kudrna D, Braidotti M, Yu Y, Parameswaran P, Crowell MD, Wing R, Rittmann BE, KrajmalnikBrown R (2009) Human gut microbiota in obesity and after gastric bypass. Proc Natl Acad Sci U S A 106(7):2365-2370. doi:10.1073/ pnas.0812600106

Zuin A, Carmona M, Morales-Ivorra I, Gabrielli N, Vivancos AP, Ayte J, Hidalgo E (2010) Lifespan extension by calorie restriction relies on the sty1 map kinase stress pathway. EMBO J 29 (5):981-991. doi:10.1038/emboj.2009.407 